\title{
The BET inhibitor CPI203 promotes ex vivo expansion of cord blood long-term repopulating HSCs and megakaryocytes
}

\author{
Tracking no: BLD-2020-005357R2
}

Peng Hua (University of Oxford, United Kingdom) Joanna Hester (University of Oxford, United Kingdom) George Adigbli (University of Oxford, United Kingdom) Rong Li (MRC Weatherall Institute of Molecular Medicine, United Kingdom) Bethan Psaila (MRC Weatherall Institute of Molecular Medicine, United Kingdom) Anindita Roy (University of Oxford, United Kingdom) Carole Bataille (University of Oxford, United Kingdom) Graham Wynne (University of Oxford, United Kingdom) Thomas Jackson (University of Oxford, United Kingdom) Thomas Milne (University of Oxford, United Kingdom) Angela Russell (Uniersity of Oxford, United Kingdom) James Davies (University of Oxford, United Kingdom) Irene Roberts (Oxford University, United Kingdom) Fadi Issa (University of Oxford, United Kingdom) Suzanne Watt (University of Adelaide, Australia)

\begin{abstract}
:
Although cytokine-mediated expansion of human hematopoietic stem cells (HSCs) can result in high yields of hematopoietic progenitor cells, this generally occurs at the expense of reduced bone marrow HSC repopulating ability thereby limiting potential therapeutic applications. As Bromodomain-containing proteins (BCPs) have been demonstrated to regulate mouse HSC self-renewal and stemness, we screened small molecules targeting various BCPs as potential agents for ex vivo expansion of human HSCs. Of 10 compounds tested, only the Bromodomain and Extra-terminal Motif (BET) inhibitor CPI203 enhanced the expansion of human cord blood HSCs without losing cell viability in vitro. The expanded cells also demonstrated improved engraftment and repopulation in serial transplantation assays. Transcriptomic and functional studies showed that the expansion of long-term repopulating HSCs was accompanied by synchronized expansion and maturation of megakaryocytes consistent with CPI203-mediated reprogramming of cord blood hematopoietic stem and progenitor cells (HSPCs). This approach may therefore prove beneficial for ex vivo gene editing, for enhanced platelet production, and for the improved usage of cord blood for transplantation research and therapy.
\end{abstract}

Conflict of interest: COI declared - see note

COI notes: PH, JH, GA, RL, BP, ARoy, CB, GW, TJ, IR, FI, SMW declare no competing financial interests. JD is a co-founder of Nucleome Therapeutics Ltd. to which he provides consultancy. TAM is a founding and minor $(<5 \%)$ shareholder of OxStem Oncology (OSO), a subsidiary company of OxStem Ltd. AR is a founding and minor $(<5 \%)$ shareholder of OxStem Ltd.

\section{Preprint server: No;}

Author contributions and disclos ures: PH conceived, designed, performed, analyzed experiments, performed bioinformatics analysis and wrote the manuscript. JH, GA and FI performed in vivo experiments. RL and BP performed the MK analysis. TJ contributed to the bioinformatics analysis. AR, CB and GW supplied chemicals and contributed to experimental analysis. SMW, FI, AR and TAM designed and supervised the project, SMW, FI, AR, TAM, ARoy, IR, JD and BP analyzed experiments and contributed to writing the manuscript. All authors reviewed and agreed the final submitted manuscript.

Non-author contributions and disclosures: No;

Agreement to Share Publication-Related Data and Data Sharing Statement: public deposit, Sequencing data has been submitted to the NCBI Gene Expression Omnibus (GSE140813).

\section{Clinical trial regis tration information (if any):}


Figure.1

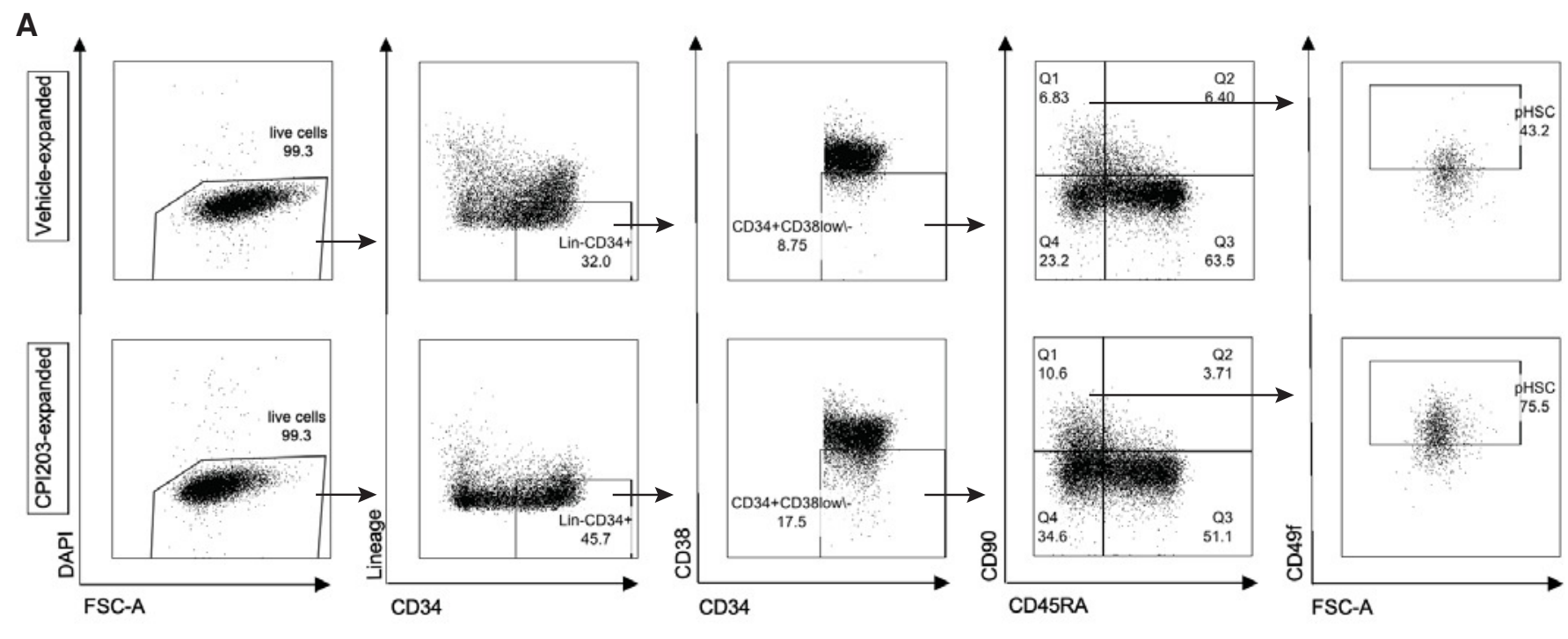

B

pHSC

Lin-CD34+CD38-CD45RA-CD90+CD49f+

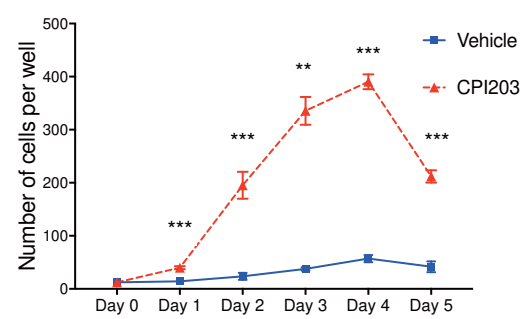

E

Unexpanded or 5-days expanded CB CD133+ cells

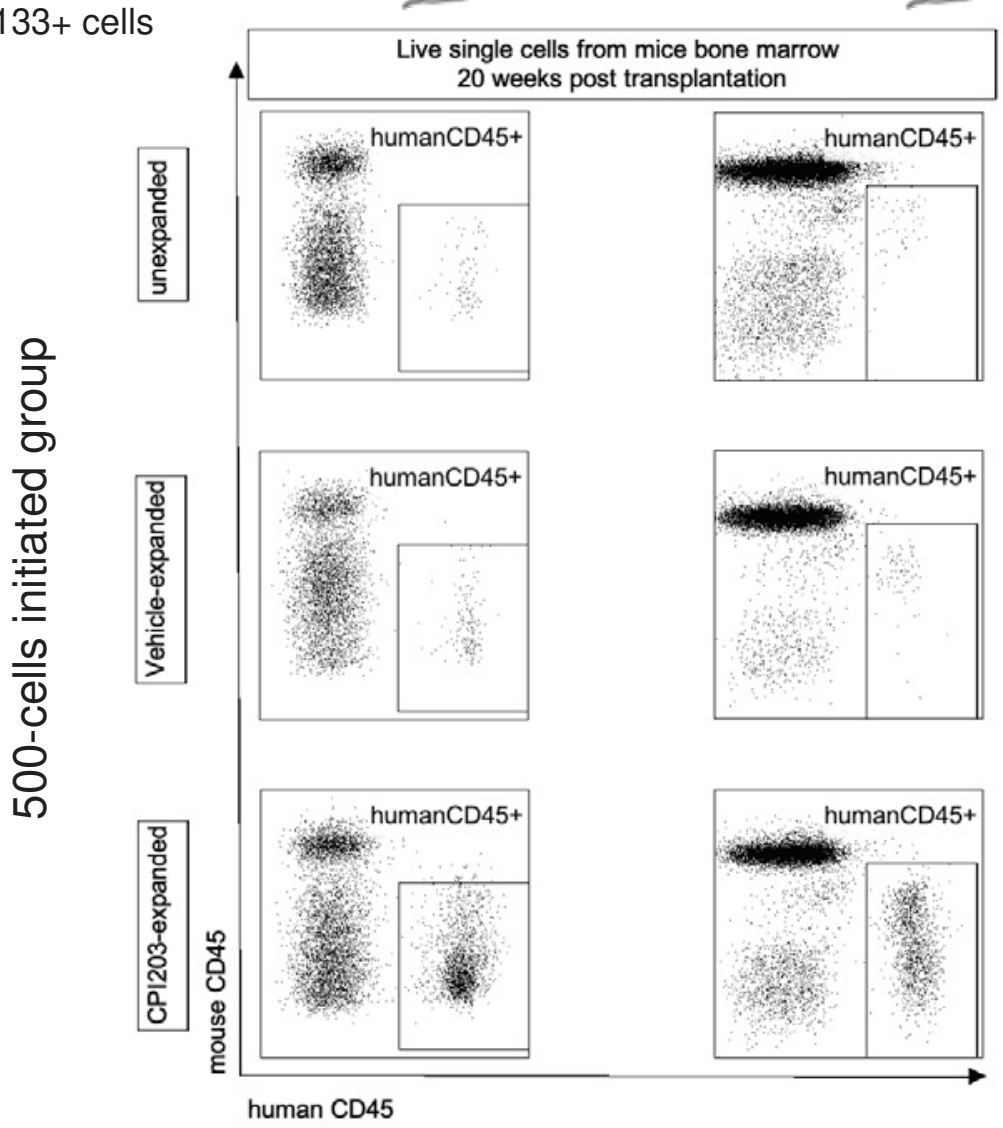

Estimated LTC-IC in pHSC

Secondary

Transplantation

Transplantation

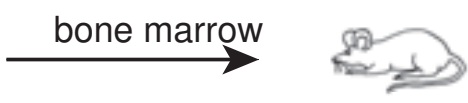

D

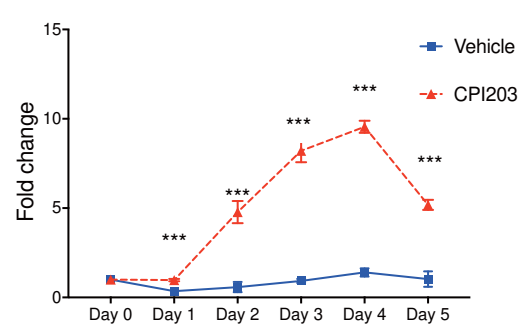

$\mathbf{F}$
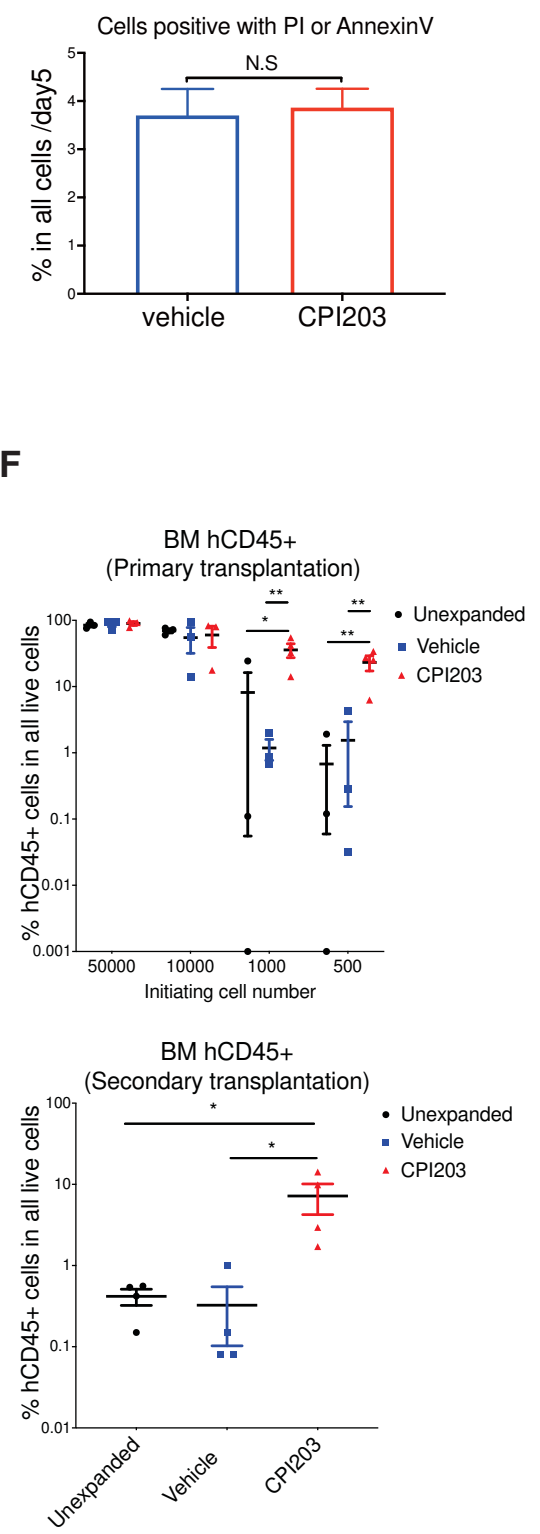
Figure.2

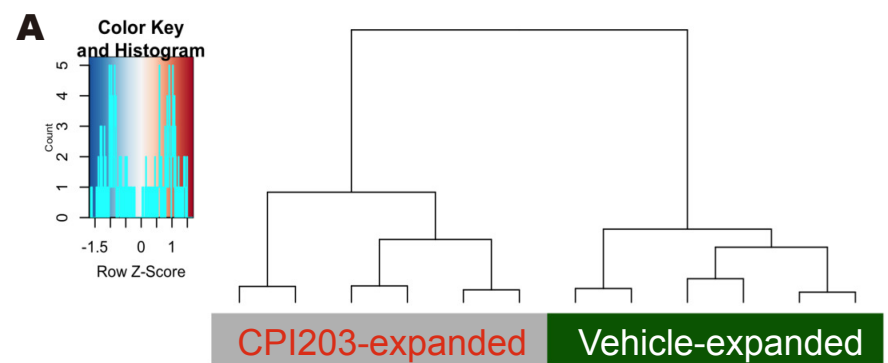

Megakaryocyte

Myeloid

Lymphoid

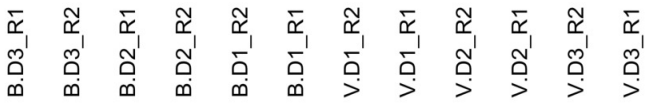

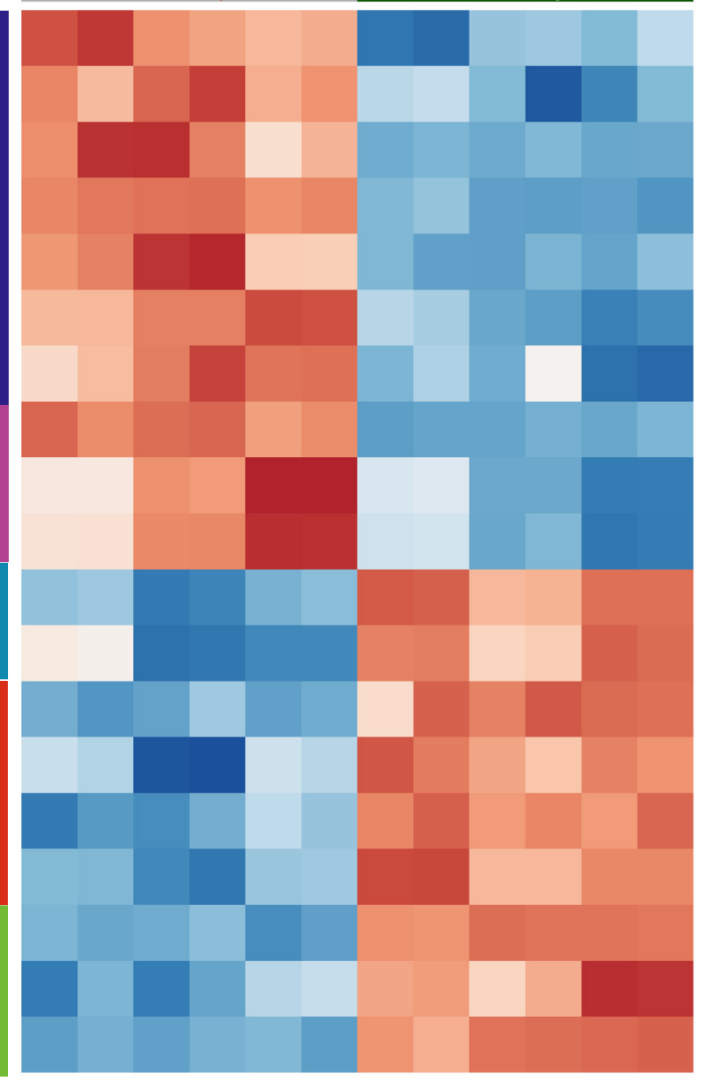

B

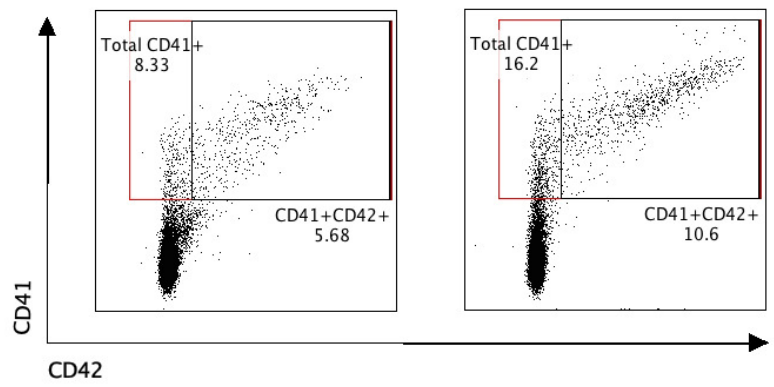

POU5F1

EMCN

CRHBP

HLF

MEIS1

JUN

CXCR4

PF4

C6orf25

CSF3R

PRTN3

CA1

CNRIP1

APOC1

TFR2

CD4

CD79A

CD79B

D

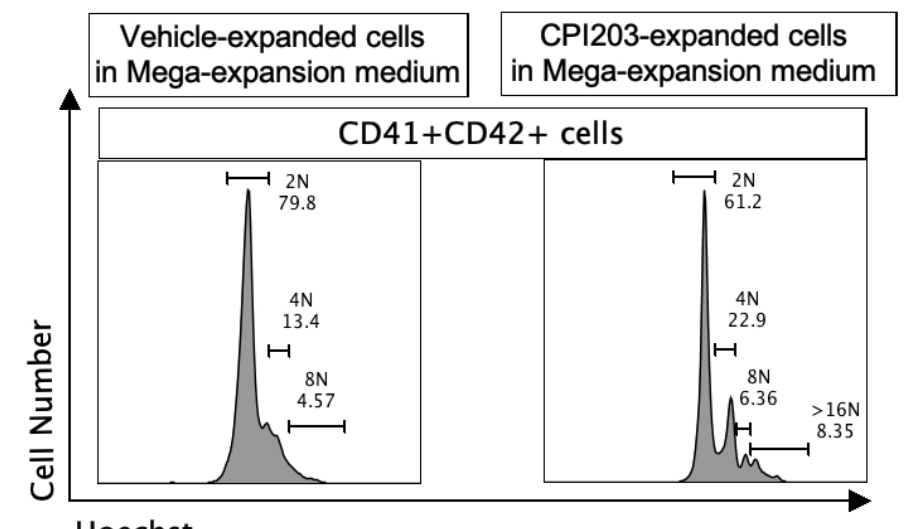

Hoechst
Vehicle/cytokines

CPI203/cytokines

C
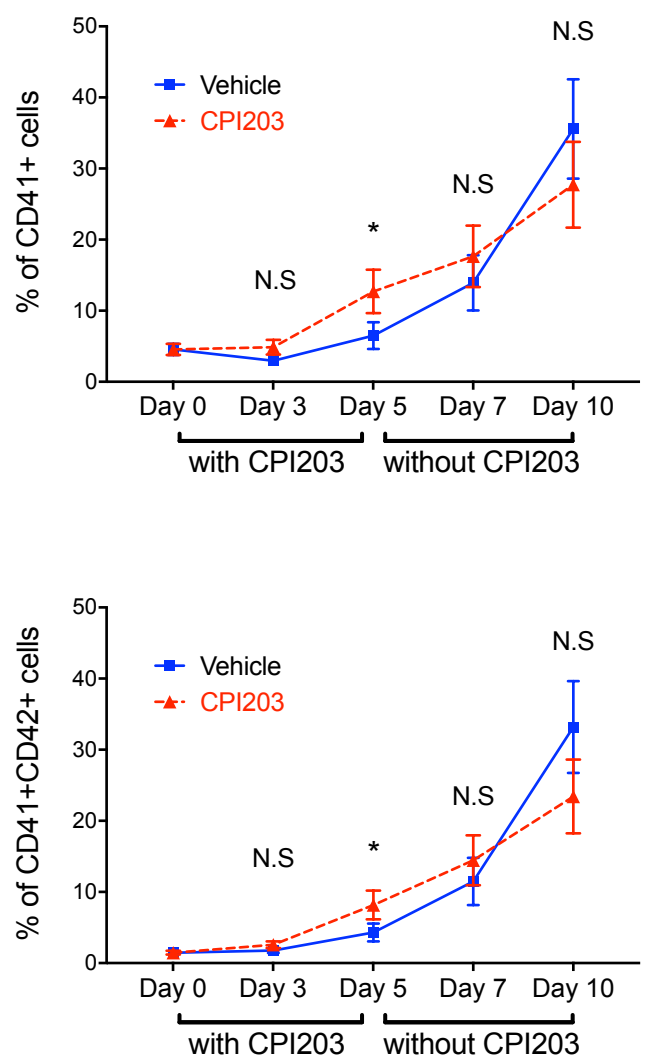

E

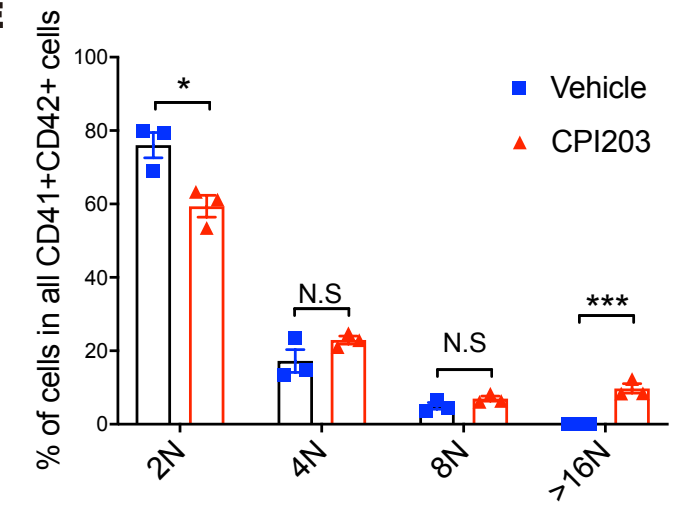


Full title:

\section{The BET inhibitor CPI203 promotes ex vivo expansion of cord blood long-term}

\section{repopulating HSCs and megakaryocytes}

Peng Hua ${ }^{1,2 *}$, Joanna Hester ${ }^{3 *}$, George Adigbli ${ }^{3 *}$, Rong $\mathrm{Li}^{1}$, Bethan Psaila ${ }^{1}$, Anindita Roy ${ }^{4}$, Carole J.R. Bataille ${ }^{5}$, Graham M. Wynne ${ }^{5}$, Thomas Jackson ${ }^{4}$, Thomas A. Milne ${ }^{1}$, Angela J. Russell $^{5,6}$, James Davies ${ }^{1,2}$, Irene Roberts ${ }^{1,4}$, Fadi Issa ${ }^{3 \#}$, Suzanne M. Watt ${ }^{2,7 \#}$

1. MRC Molecular Haematology Unit, Weatherall Institute of Molecular Medicine, Radcliffe Department of Medicine, John Radcliffe Hospital, Oxford, OX3 9DS, UK.

2. Nuffield Division of Clinical Laboratory Medicine, Radcliffe Department of Medicine, University of Oxford, John Radcliffe Hospital, Oxford, OX3 9BQ, UK.

3. Transplantation Research Immunology Group, Nuffield Department of Surgical Sciences, University of Oxford, John Radcliffe Hospital, Oxford, OX3 9DU, UK.

4. Department of Paediatrics, University of Oxford, Children's Hospital, John Radcliffe Hospital, Oxford OX3 9DU, UK.

5. Department of Chemistry, University of Oxford, Chemistry Research Laboratory, Mansfield Road, Oxford OX1 3TA, UK.

6. Department of Pharmacology, University of Oxford, Mansfield Road, Oxford OX1 3QT, UK.

7. Adelaide Medical School, Faculty of Health and Medical Sciences, University of Adelaide, Adelaide 5005, and South Australian Health and Medical Research Institute, Adelaide 5001, Australia.

* These authors contributed equally. \# These senior authors contributed equally.

Short title: CPI203 expanded UCB LT-HSCs and megakaryocytes

\section{Address for correspondence:}

Peng Hua Ph.D. and Suzanne M. Watt Ph.D., FRCPath., Weatherall Institute of Molecular Medicine /Nuffield Division of Clinical Laboratory Medicine, Radcliffe Department of Medicine, John Radcliffe Hospital, Oxford, OX3 9DS, UK.

Tel: +441865222419

Email address: peng.hua@ndcls.ox.ac.uk and suzanne.watt@ndcls.ox.ac.uk

\section{Word counts:}

Text: max. 1200; currently 1304 words Abstract: max. 200; currently 169 words

Figure count: max. 2; currently 2

Reference count: max. 25; currently 21

\section{Contact details:}

Peng Hua: peng.hua@ndcls.ox.ac.uk Joanna Hester: joanna.hester@nds.ox.ac.ok 
George Adigbli: george.adigbli@nds.ox.ac.uk Rong li: rong.li@ndcls.ox.ac.uk

Bethan Psaila: bethan.psaila@ndcls.ox.ac.uk Anindita Roy: anindita.roy@paediatrics.ox.ac.uk Carole J.R. Bataille: carole.bataille@chem.ox.ac.uk Graham M. Wynne: graham.wynne@yahoo.co.uk Thomas Jackson: thomas.jackson@paediatrics.ox.ac.uk Thomas A. Milne: thomas.milne@imm.ox.ac.uk Angela J. Russell: angela.russell@chem.ox.ac.uk James Davies: james.davies@ndcls.ox.ac.uk Irene G. Roberts: irene.roberts@paediatrics.ox.ac.uk Fadi Issa: fadi.issa@nds.ox.ac.uk

Suzanne M. Watt: suzanne.watt@ndcls.ox.ac.uk 


\section{Key Points:}

1. CPI203-expanded cord blood cells retain bone marrow repopulating capacity.

2. CPI203 promotes human megakaryocyte development ex vivo.

\section{Abstract:}

Although cytokine-mediated expansion of human hematopoietic stem cells (HSCs) can result in high yields of hematopoietic progenitor cells, this generally occurs at the expense of reduced bone marrow HSC repopulating ability thereby limiting potential therapeutic applications. As Bromodomain-containing proteins (BCPs) have been demonstrated to regulate mouse HSC self-renewal and stemness, we screened small molecules targeting various BCPs as potential agents for ex vivo expansion of human HSCs. Of 10 compounds tested, only the Bromodomain and Extra-terminal Motif (BET) inhibitor CPI203 enhanced the expansion of human cord blood HSCs without losing cell viability in vitro. The expanded cells also demonstrated improved engraftment and repopulation in serial transplantation assays. Transcriptomic and functional studies showed that the expansion of long-term repopulating HSCs was accompanied by synchronized expansion and maturation of megakaryocytes consistent with CPI203-mediated reprogramming of cord blood hematopoietic stem and progenitor cells (HSPCs). This approach may therefore prove beneficial for ex vivo gene editing, for enhanced platelet production, and for the improved usage of cord blood for transplantation research and therapy.

\section{Key words:}

BET Inhibitors; human cord blood; in vitro HSC expansion; megakaryocytes; in vivo repopulation. 


\section{Introduction}

Gene regulation through epigenetic modification is vital for normal and malignant hematopoiesis ${ }^{1}$, and epigenetic regulators have been highlighted as important therapeutic targets. Small molecules that target chromatin-regulating proteins, such as histone deacetylase (HDAC) inhibitors and DNA demethylating agents, overcome the loss-ofstemness by human hematopoietic stem cells (HSCs) that occurs in ex-vivo expansion cultures, but it is unclear if all cell types can be generated by reported protocols, especially megakarocytes ${ }^{2-4}$. Bromodomain-containing proteins (BCPs), as components of transcription factor complexes and determinants of epigenetic memory ${ }^{1,5,6}$, have been implicated in regulating mouse HSC self-renewal and 'stemness ${ }^{17,8}$, but are not well-studied in normal human hematopoiesis. By screening small molecules that target various BCPs in umbilical cord blood (UCB) HSCs, we demonstrate that the Bromodomain and Extra-terminal Motif (BET) domain inhibitor CPI203 promotes ex vivo expansion of long-term repopulating human HSCs and megakaryocytes.

\section{Methods}

CD133+ human UCB cells (purity $>90 \%$ ) were cultured under optimised conditions for five days in stem cell expansion cultures (with SCF, TPO and FLT3L as described ${ }^{4,9-11}$ ). Detailed methods are described in Supplemental Materials. Sequencing data have been submitted to the NCBI Gene Expression Omnibus (GSE140813). 


\section{Results and Discussion}

\section{BET inhibitors support ex-vivo expansion of phenotypic HSCs}

To investigate the role of $\mathrm{BCPs}$ in normal hematopoiesis, we tested $10 \mathrm{BCP}$ inhibitors targeting BRD2-4, BRDT, BAZ2A/B, CREBBP, EP300, SMARCA or PB1 on UCB CD133+ cells (Supplemental Figure 1A). Cells were plated in stem cell expansion media with increasing concentrations of these compounds (60nM to $15 \mu \mathrm{M})$, then analyzed by flow cytometry (Supplemental Table 1). Lin-CD34+CD38-CD45RA-CD90+CD49f+ cells were identified phenotypically as HSCs (pHSC) $)^{12}$. Only BET inhibitors resulted in increased absolute numbers of pHSCs (Supplemental Figure 1B), consistent with previous studies demonstrating increased LSK cells/HSCs in mice administered with JQ1 ${ }^{8}$. Most (JQ1, PFI-1, Bromosporine, OXF-BD-02; Supplemental Figure 1B) showed their greatest effects at concentrations $>5000 \mathrm{nM}$, which also reduced cell viability. In contrast, with CPI203, the numbers of pHSC increased significantly, commencing from concentrations as low as $60 \mathrm{nM}$, with cells remaining viable up to 540nM (Supplemental Figure 1B).

To further assess CPI203, 5000 UCB CD133+ cells/well were cultured in cytokines with 150nM CPI203 for 5 days (Figure 1A). While total nucleated cells (TNC) steadily increased in the vehicle/cytokine control cultures as expected, due mainly to an expansion of immunophenotypic LMPP(Lin-CD34+CD38-CD45RA+) and Lin+ compartments, TNC expansion was significantly less in the CPI203/cytokines cultures than in vehicle/cytokines (Supplemental Figure 2A). Furthermore, numbers of total Lin-CD34+ HSPCs were not altered by addition of CPI203 compared to the vehicle/cytokine control ( $p=0.90$ at day 5 ) and cell viability was unaffected by CPI203 treatment over time ( $p=0.82$ at day 5 ; Supplemental Figure 2A). However, the absolute number of pHSC started to increase significantly by 24 hours after culture initiation with $\mathrm{CPI} 203$ and remained significantly higher than the vehicle/cytokine control in the following 4 day culture (Figure 1B). Similarly, the increase in absolute numbers of immunophenotypic MPP (Lin-CD34+CD38-CD45RA-CD90-) occurred earlier in CPI203 than vehicle-containing cultures (Supplemental Figure 2A). 
To estimate the proportion of Long-Term Culture-Initiating Cells (LTC-IC) in the pHSC subset, we performed a limiting-dilution-analysis (LDA) on pHSCs sorted pre and post 5days of expansion by CPI203/cytokines or vehicle/cytokines. LTC-IC frequency was almost identical (1-in-42.4 vs 1 -in-42.2 for day 5 cells for day 5 CPI203-treated and vehicle-treated pHSCs respectively; Supplemental Figure 2B). However, as the absolute number of pHSCs in CPI203/cytokine cultures was 5 to10-fold higher than the vehicle/cytokine control from day 2 of culture (Figure 1B), and cell viability was unaffected by CPI203 (Figure 1D, $p=0.67$ Supplemental Figure 2C), we estimate that CPI203 expanded absolute numbers of LTC-ICs 5-10 fold compared to a minimal expansion by vehicle/cytokines (Figure 1C, $p<0.001, n=4$ ). As expected, while the LTC-IC frequency was higher in unexpanded pHSC (1-in-12.9), consistent with the pHSC frequency $(0.1 \pm 0.2 \%$ in CD133+ cells $)$ and similar to that observed in previous repopulation studies using irradiated NSG mice ${ }^{12}$, the total numbers of LTC-IC in the pHSC fraction of CPI203 cultures were 1.5-3 times higher from days 2-5 compared to the unexpanded pHSC.

\section{CPI203/cytokines-expanded CD133+ cells show improved bone marrow (BM)

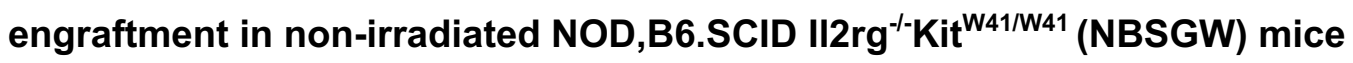
To confirm preservation of HSC function of CPI203-expanded cells in vivo, unexpanded CD133+ cells $(500-50,000)$, or the total progeny of that cell number after expansion, were injected into non-irradiated adult NBSGW mice (Supplemental Table 2). After 20-22 weeks, all 14 mice injected with CPI203/cytokines-expanded cells engrafted the BM $(\geq 1 \%$ human (h)CD45+ cells) at all cell doses, while consistent engraftment of vehicle/cytokine-expanded cells only occurred with higher cell doses (Figure 1E-F, Supplemental Tables 2-4). Indeed, the level of BM engraftment in the 500 -cell initiating group was $23.1 \% \pm 5.9 \%$ in CPI203/cytokine conditions vs $0.7 \% \pm 0.6 \%$ for vehicle/cytokines (Figure 1E-F). Multilineage reconstitution was observed, with substantial hCD235a+ erythroid cells $(40.1 \% \pm 15.2 \%)$, as well as lymphocytes (CD19+ B-cells: $20.1 \% \pm 5.9$; CD3+ T-cells: $1.4 \% \pm 1.2 \%$ ), CD33+ 
myeloid cells $(0.7 \% \pm 0.2 \%)$, and Lin-CD34+ HSPCs $(0.2 \% \pm 0.1 \%)$, detected in the total BM of engrafted mice (Supplemental Figure 3A-F, Supplemental Tables 3 and 4). Spleen and peripheral blood (PB) engraftment with hCD45+ cells were also higher for CPI203/cytokine expanded cells in 500 -cell initiating group: spleen- $37.6 \% \pm 13.2 \%$ vs. $4.5 \% \pm 3.3 \%$; PB$5.8 \% \pm 3.2 \%$ vs. $0.2 \% \pm 0.2 \%$ ) (Supplemental Figure 4 A-C. See all data in other cell dosage in Supplemental Figure 4 and Supplemental Table 4).

To confirm long-term repopulating capacity of CPI203/cytokine-treated cells, secondary transplantation was performed and analyzed 22 weeks after injection. All mice injected with BM cells from mice receiving CPI203/cytokine-expanded cells engrafted $(7.2 \% \pm 2.9 \%$ hCD45+ cells in $\mathrm{BM}$ ) with multilineage reconstitution, compared to $0.3 \% \pm 0.2 \%$ hCD $45+$ cells in mice transplanted with the same number of BM cells from mice receiving vehicle/cytokineexpanded cells (Figure 1E-F, Supplemental Figure 5-6, Supplemental Table 5) confirming that CPI203 can expand phenotypic and functional LT-HSC.

\section{CPI203 promotes expansion of HSC and megakaryocytes}

Finally, to investigate the transcriptomic effects of CPI203 on UCB HSPCs, we performed RNA sequencing on cells expanded with CPI203/cytokines or vehicle/cytokines. Differences in expression of many HSC-related genes were detected (Figure 2A, Supplemental Figure 7A-B and Supplemental Excel Sheets). Genes highly expressed in HSCs (PROM1, POU5F1, EMCN, CRHBP, HLF, MEIS1, JUN and CXCR4 ${ }^{2,9,13-16}$ ) were significantly more highly expressed in CPI203/cytokine-expanded cells, in keeping with the higher numbers of pHSCs. In contrast, myeloid, erythroid and lymphoid-related genes ${ }^{13}$, including CSF3R, PRTN3, CA1, CNRIP1, APOC1, TFR2, CD4, CD79A and CD79B, were more highly expressed in vehicle/cytokine conditions. Unexpectedly, three of the most upregulated genes in CPI203/cytokine-expanded cells are strongly associated with megakaryocyte (MK) development (CXCR4 ${ }^{17}, \mathrm{PF} 4^{18}$ and $\mathrm{C} 6$ orf $\left.25^{19}\right)$. 
We therefore examined the percentage of MK in the cultures. After expansion in CPI203/cytokines for 5 days (Figure 2B-C), the proportion of both CD41+ and CD41+CD42+ MK was significantly ( 2-fold) higher than for the vehicle/cytokine control. To further test the effect of CPI203 on MK differentiation and maturation, the day 5 CPI203/cytokines or vehicle/cytokines expanded cells were transferred into MK-supportive media (SCF, TPO) and cultured without CPI203 or vehicle for a further 5 days. The percentages of CD41+ and CD41+CD42+ MK increased in the second culture phase (Figure $2 C$ ), and, by day $10, M K$ derived from the d5-CPI203-expanded cells were of higher ploidy than the d5vehicle/cytokine-expanded UCB HSPCs (Figure 2D-E). This suggests that CPI203 promotes MK maturation, and is consistent with previous work showing that BET inhibition promotes enlargement, polyploidization and proplatelet formation of human UCB MK-progenitors ${ }^{20}$. Whether BET inhibition might also exert effects through reprogramming towards MK-biased HSCs is an interesting and pertinent question, but further work would be needed to address this.

In summary, we demonstrate that the BET inhibitor CPI203 expands serially transplantable LT-HSCs ex-vivo and, in contrast to UM171, which inhibits erythroid and megakaryocytic differentiation during ex-vivo expansion of LT-HSCs ${ }^{21}$, CPI203 also supports megakaryocyte maturation. CPI203 may therefore prove beneficial for enhanced platelet production and for the improved usage of UCB for transplantation. Understanding the mechanisms by which CPI203 exerts these effects should provide fundamental insight into MK development. 


\section{Acknowledgements:}

These studies received grant support from an MRC Discovery Award led by Professor Doug Higgs (PH, JH, GA, AR, JD, TAM, IR, FI, SMW. Ref: MC_PC_15069). JD and PH are also funded by an MRC Clinician Scientist Award (MRC Clinician Scientist Fellowship ref. MR/R008108) to JD. JH is a recipient of KRUK Senior Fellowship, GA is a recipient of Clarendon Scholarship, FI and ARoy are both recipients of Wellcome Trust CRCD Fellowships. RL is funded by a Bloodwise project grant and BP is a recipient of Cancer Research UK Advanced Clinician Scientist Fellowship. TAM was funded by Medical Research Council (MRC, UK) Molecular Haematology Unit grant MC_UU_12009/6 and is supported by the NIHR Oxford Biomedical Research Centre Haematology Theme.

\section{Authorship Contributions:}

$\mathrm{PH}$ conceived, designed, performed, analyzed experiments, performed bioinformatics analysis and wrote the manuscript. JH, GA and FI performed in vivo experiments. RL and BP performed the MK analysis. TJ contributed to the bioinformatics analysis. AR, CB and GW supplied chemicals and contributed to experimental analysis. SMW, FI, AR and TAM designed and supervised the project, SMW, FI, AR, TAM, ARoy, IR, JD and BP analyzed experiments and contributed to writing the manuscript. All authors reviewed and agreed the final submitted manuscript.

\section{Conflict-of-interest disclosure:}

$\mathrm{PH}, \mathrm{JH}, \mathrm{GA}, \mathrm{RL}, \mathrm{BP}, \mathrm{ARoy}, \mathrm{CB}, \mathrm{GW}, \mathrm{TJ}, \mathrm{IR}, \mathrm{FI}, \mathrm{SMW}$ declare no competing financial interests. JD is a co-founder of Nucleome Therapeutics Ltd. to which he provides consultancy. TAM is a founding and minor ( $<5 \%)$ shareholder of OxStem Oncology (OSO), a subsidiary company of OxStem Ltd. AR is a founding and minor $(<5 \%)$ shareholder of OxStem Ltd. 


\section{References:}

1. Jones PA, Issa J-PJ, Baylin S. Targeting the cancer epigenome for therapy. Nature Rev Genet. 2016;17(10):630-641.

2. Chaurasia P, Gajzer DC, Schaniel C, D'Souza S, Hoffman R. Epigenetic reprogramming induces the expansion of cord blood stem cells. J Clin Invest. 2014;124(6):2378-2395.

3. Milhem M, Mahmud N, Lavelle D, et al. Modification of hematopoietic stem cell fate by 5aza 2'deoxycytidine and trichostatin A. Blood. 2004;103(11):4102-4110.

4. Hua P, Kronsteiner B, Ashley N, et al. Molecular insights at the single cell level into the reprogramming of human hematopoietic stem cells during epigenetically modified expansion [abstract]. Blood. 2017;130(Suppl 1):2425.

5. Shi J, Vakoc CR. The mechanisms behind the therapeutic activity of BET bromodomain inhibition. Mol Cell. 2014;54(5):728-736.

6. Crump NT, Ballabio E, Godfrey L, et al. BET inhibition disrupts transcription but retains enhancer-promoter contact. bioRxiv. 2019:848325.

7. Dey A, Yang W, Gegonne A, et al. BRD4 directs hematopoietic stem cell development and modulates macrophage inflammatory responses. EMBO J. 2019;38(7).

8. Wroblewski M, Scheller-Wendorff M, Udonta F, et al. BET-inhibition by JQ1 promotes proliferation and self-renewal capacity of hematopoietic stem cells. Haematologica. 2018;103(6):939-948.

9. Hua P, Kronsteiner B, van der Garde M, et al. Single-cell assessment of transcriptome alterations induced by Scriptaid in early differentiated human haematopoietic progenitors during ex vivo expansion. Scientific Reports. 2019;9(1):5300.

10. Tarunina M, Hernandez D, Kronsteiner-Dobramysl B, et al. A novel high-throughput screening platform reveals an optimized cytokine formulation for human hematopoietic progenitor cell expansion. Stem Cells Dev. 2016;25(22):1709-1720.

11. Gullo F, Van Der Garde M, Russo G, et al. Computational modeling of the expansion of human cord blood CD133+ hematopoietic stem/progenitor cells with different cytokine combinations. Bioinformatics. 2015;31(15):2514-2522.

12. Notta F, Doulatov S, Laurenti E, Poeppl A, Jurisica I, Dick JE. Isolation of single human hematopoietic stem cells capable of long-term multilineage engraftment. Science. 2011;333(6039):218-221.

13. Hua P, Roy NBA, de la Fuente J, et al. Single cell analysis of bone marrow derived CD34+ cells from children with sickle cell disease and thalassemia. Blood. 2019.

14. Nie Y, Han Y-C, Zou Y-R. CXCR4 is required for the quiescence of primitive hematopoietic cells. J Exp Med. 2008;205(4):777.

15. Sugiyama T, Kohara H, Noda M, Nagasawa T. Maintenance of the hematopoietic stem cell pool by CXCL12-CXCR4 chemokine signaling in bone marrow stromal cell niches . Immunity. 2006;25(6):977-988.

16. Corces MR, Buenrostro JD, Wu B, et al. Lineage-specific and single-cell chromatin accessibility charts human hematopoiesis and leukemia evolution. Nature Genet.

2016;48(10):1193-1203.

17. Wang JF, Liu ZY, Groopman JE. The alpha-chemokine receptor CXCR4 is expressed on the megakaryocytic lineage from progenitor to platelets and modulates migration and adhesion. Blood. 1998;92(3):756-764.

18. Psaila B, Wang G, Meira AR, et al. Single-cell analyses reveal aberrant pathways for megakaryocyte-biased hematopoiesis in myelofibrosis and identify mutant clone-specific targets. bioRxiv. 2019:642819.

19. Psaila B, Barkas N, Iskander D, et al. Single-cell profiling of human megakaryocyteerythroid progenitors identifies distinct megakaryocyte and erythroid differentiation pathways. Genome Biol. 2016;17(1):83.

20. Elagib KE, Lu CH, Mosoyan G, et al. Neonatal expression of RNA-binding protein IGF2BP3 regulates the human fetal-adult megakaryocyte transition. J Clin Invest.

2017;127(6):2365-2377. 
21. Tajer P, Pike-Overzet K, Arias S, Havenga M, Staal FJT. Ex Vivo Expansion of Hematopoietic Stem Cells for Therapeutic Purposes: Lessons from Development and the Niche. Cells. 2019;8(2).

Figure Legends:

Figure 1. Cord blood hematopoietic stem/progenitor cell expansion ex-vivo by the BET inhibitor CPI203

A) Flow plots of 5-day expanded UCB CD133+ cells in vehicle/cytokines (top) and CPI203/cytokines (bottom), showing the expression of Lineage (Lin), CD34, CD38, CD45RA, CD90 and CD49f markers. Sequential gating strategy from left to right. B) Absolute numbers of phenotypically-defined (p) Lin-CD34+CD38-CD45RA-CD90+CD49f+ HSCs increased significantly in CPI203/cytokine condition compared to the vehicle/cytokine control (**, $p \leq 0.01 ;{ }^{* * *}, p \leq 0.005$; each well seeded with 5000 cells, $\left.n=4\right)$. C) Absolute number of LTC-IC per well in 5 day CPI203/cytokine vs vehicle/cytokine expansion cultures based on LDA estimation. ( $\left.\mathrm{n}=4,{ }^{* *}, p \leq 0.01 ;{ }^{* *}, p \leq 0.005\right)$. D) Percentage of Annexin V+PI- (apoptotic) and Annexin V+PI+ (dead) cells in cytokine containing medium supplemented with $\mathrm{CPI} 203$ or the vehicle control did not differ on day 5 of expansion $(n=3, N S p=0.82$. E) Bone marrow cells harvested from mice injected with 500 unexpanded UCB CD133+ cells or progeny of 500 culture initiating UCB CD133+ cells show significantly more human cells in CPI203/cytokineexpanded conditions than unexpanded or vehicle/cytokines conditions (left: primary transplantation; right: secondary transplantation, all replicates shown in Supplemental Figure 3-6 and Table 2-5). F) Dot plots show the percentage of hCD45+ human leukocytes in all bone marrow cells from recipient mice in different conditions for (top) primary transplants and (bottom) secondary transplants $\left(n=3-4 ;{ }^{*}, p \leq 0.05 ;{ }^{* *}, p \leq 0.01\right.$, One-way ANOVA with multiple comparison (Fisher's LSD)).

Figure 2. CPI203 promotes megakaryocyte-accompanied ex-vivo expansion of $\mathrm{CB}$ HSCs 
A) RNA sequencing was performed on UCB CD133+ cells which had been expanded for 5days in culture with CPI203/cytokines (B) or vehicle/cytokines (V). Top differentially expressed genes associated with HSCs, megakaryocytes, myeloid cells, erythroid cells and lymphoid cells are shown in a heatmap (FDR $<0.01$, gene expression level from low to high shown as blue to orange). B) Flow plots showing the total CD41+ or CD41+CD42+ cells detected in all cells expanded in CPI203/cytokines condition compared to vehicle/cytokines on day 5. C) Increased total CD41+ (top) and CD41+CD42+ (bottom) megakaryocytic cells expanded in CPI203/cytokine conditions compared to vehicle/cytokine for 5 days, followed by a further 5-days' expansion in MK expansion medium (TPO, SCF) without CPI203 or vehicle $\left(n=3 .{ }^{*}, p \leq 0.05\right)$. D-E) Increased ploidy of megakaryocytes generated in MK expansion medium from d5-CPI203/cytokine-expanded cultures compared to d5vehicle/cytokine-expanded cultures $\left(n=3 ;{ }^{*}, p \leq 0.05 ;{ }^{* * *}, p \leq 0.005\right)$. Data shown are from day 10 of extended cultures: 5 days in CPI203/cytokines or vehicle/cytokines followed by 5 days in MK expansion medium (SCF, TPO) without CPI203 or vehicle. 